\title{
Prospects for High Resolution Analytical Electron Microscopy of Organic Crystalline Particles.
}

\author{
James Cattle ${ }^{1}$, Mark S'ari ${ }^{1}$, Neil Wilkinson ${ }^{2}$, Nicole Hondow ${ }^{1}$, Andy Brown ${ }^{1}$ and Rik Brydson ${ }^{1}$. \\ 1. Institute for Materials Research, School of Chemical and Process Engineering, University of Leeds, \\ Leeds LS2 9JT, United Kingdom. \\ 2. Gatan UK, 25 Nuffield Way, Abingdon, Oxon. OX14 1RL, United Kingdom.
}

Polymorphism is the ability of a compound to crystallise with different packing arrangements and is critically important in several industrial sectors as many of the solid-state properties of a compound are dependent on the crystal form. Recently, TEM has been applied to the study of polymorphism in organic materials such as pharmaceuticals, where polymorphs are extremely common, and has been shown to have advantages over more routinely used analytical techniques [1]. Using a combination of imaging and diffraction each of the crystals in a sample can be analysed one-by-one during TEM analysis and different polymorphs of a compound can be distinguished, or new polymorphs of a compound identified, on the basis of crystal morphology and/or by indexing electron diffraction patterns. A further advantage is the ability to solve crystal structures of beam sensitive organic compounds using a single crystallite with sub-micron sized dimensions, by combining electron diffraction and crystal structure prediction, where standard single crystal or powder X-ray approaches would not be applicable [2].

In addition to electron diffraction, chemical analysis at the single particle level using electron energy loss spectroscopy (EELS) is beneficial not only to identify light element stoichiometries in organic crystals, but also to identify polymorphic variants using electron energy loss near edge structure (ELNES). Direct high resolution diffraction and even phase contrast imaging could allow identification of particle distributions within complex particulate product mixtures, as well as defects within individual particles and surface facets arising as a result of powder processing or tableting requirements. This could affect overall product properties, such as the dissolution of active pharmaceutical ingredients.

A major issue preventing such analysis in organic crystalline materials is electron beam induced damage. Hence, prior to any investigation, a detailed study of both the critical fluence (aka "dose") and fluence rates is required in order to identify the safe limits for analysis by electron diffraction, imaging and also spectroscopy. Here we present results for a model organic compound, Theophylline, which is a xanthine derivative with the chemical formula $\mathrm{C}_{7} \mathrm{H}_{8} \mathrm{~N}_{4} \mathrm{O}_{2}$ used as a treatment for asthma and bears a structural and pharmacological similarity to caffeine $\left(\mathrm{C}_{8} \mathrm{H}_{10} \mathrm{~N}_{4} \mathrm{O}_{2}\right)$. Theophylline crystals were prepared by cooling a saturated solution in nitromethane. The nitromethane dispersion was drop cast onto either holey or continuous carbon coated (or graphene) TEM grids and analysed in a field emission TEM operated at $200 \mathrm{kV}$. Crystals were predominantly plate-like and oriented with the $\langle 100\rangle$ axis parallel to the electron beam.

Beam damage (presumed to be by a radiolytic mechanism) was monitored by observation of the diffraction pattern. Interestingly, progressive amorphisation by the electron beam proceeded via progressive loss of short range order (fading of the outer diffraction spots first) as observed by Glaeser [3] possibly due to breaking of hydrogen bonds and small molecular rearrangements during the initial stages of damage. Figure 1 shows plots of the intensity in the (011) diffraction spot of theophylline (polymorphic form II) as a function of increasing fluence, for a fluence rate of ca. $2 \times 10^{-4} \mathrm{~A} / \mathrm{cm}^{2}$. Five 
different cases are shown (details in figure caption) and it is clear that using a graphene support (and liquid nitrogen sample cooling) gave the largest critical fluence. Such analyses for a particular material can help devise appropriate measurement protocols.

At the higher fluence rates required for phase contrast imaging using conventional CCD cameras the critical fluence decreases significantly. Figure 2 shows a high resolution phase contrast image of theophylline recorded using a direct electron detection camera (Gatan K2 Summit) and a total fluence of ca. 200 electrons $/ \mathrm{nm}^{2}$ at a relatively low fluence rate of $8 \times 10^{-5} \mathrm{~A} / \mathrm{cm}^{2}$. (002) lattice fringes are visible in the raw image and more clearly in the Fourier filtered image (figure 2(b)).

EELS spectra (shown in reference [4]) were collected in diffraction mode with a collection semi-angle of 30 mrads (and typically a 20 second acquisition time) from a crystalline area of ca. 0.8 micron diameter recorded with an electron fluence rate of ca. $4 \times 10^{-5} \mathrm{~A} / \mathrm{cm}^{2}$ and a total electron fluence during spectral acquisition of typically 1500-2000 electrons $/ \mathrm{nm}^{2}$. EELS showed the presence of carbon, nitrogen and oxygen in the sample and quantification gave a $[\mathrm{C}] /[\mathrm{N}]$ atom ratio of $1.7(1.75$ is expected for theophylline) and a $[\mathrm{C}] /[\mathrm{O}]$ ratio of 2.6 (3.5 is expected for theophylline).

\section{References:}

[1] M D Eddleston et al., J. Pharm. Sci. 99 (2010), p. 4072.

[2] M D Eddleston et al., Chem. Eur. J 19 (2013), p. 7874.

[3] R M Glaeser, J. Ultrastructure Res. 36 (1971), p. 466.

[4] R Brydson et al., J. Phys.: Conf. Ser. 522 (2014), 012060.

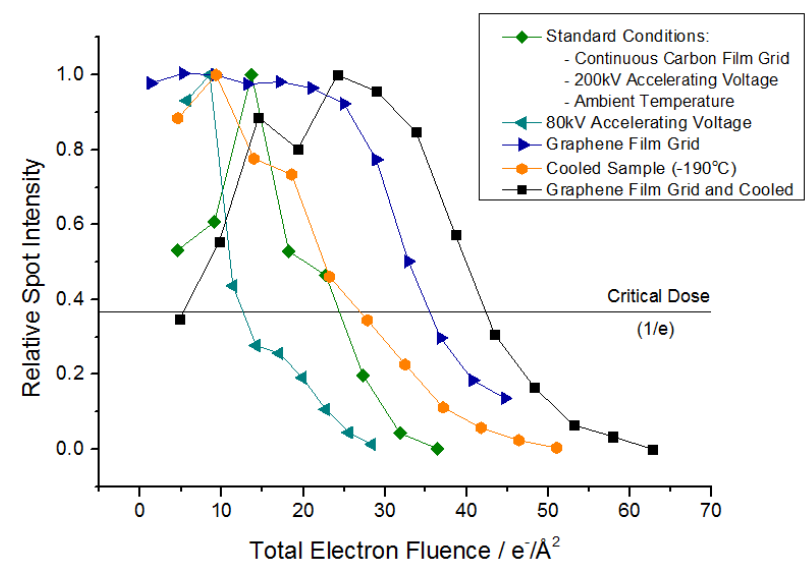

Figure 1. Graph of the decay in theophylline (011) diffraction spot intensity as a function of electron fluence for various accelerating voltages, sample support films and sample cooling.

(a)

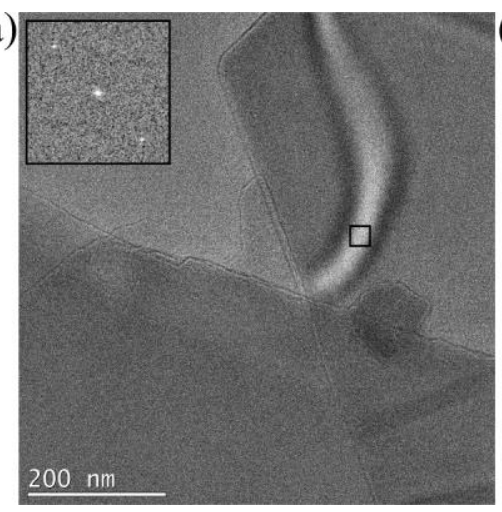

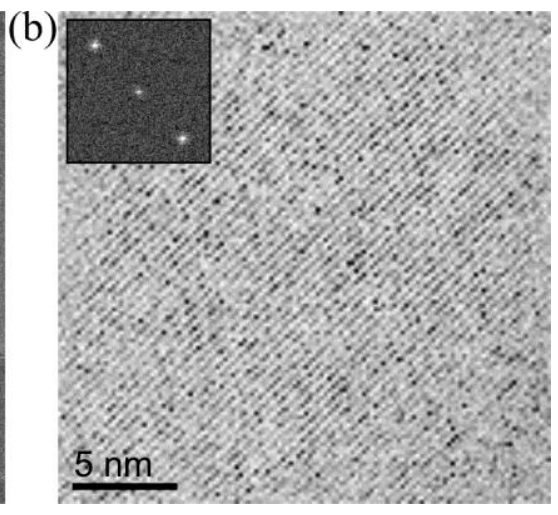

Figure 2. (a) Bright field TEM image of theophylline crystal with inset FFT from bend contour showing $0.424 \mathrm{~nm}$ (002) spots; (b) inset is the masked FFT with spots intensified by $\mathrm{x} 10$ and its inverse FFT showing lattice fringes. 